CALT-68-2204

hep-th/9812037

\title{
Introduction to M Theory and AdS/CFT Duality'
}

\author{
John H. Schwarz \\ California Institute of Technology, Pasadena, CA 91125, USA
}

\begin{abstract}
An introductory survey of some of the developments that have taken place in superstring theory in the past few years is presented. The main focus is on three particular dualities. The first one is the appearance of an 11th dimension in the strong coupling limit of the type IIA theory, which give rise to $\mathrm{M}$ theory. The second one is the duality between the type IIB theory compactified on a circle and $\mathrm{M}$ theory on a two-torus. The final topic is an introduction to the recently proposed duality between superstring theory or M theory on certain anti de Sitter space backgrounds and conformally invariant quantum field theories.
\end{abstract}

To be published in the Proceedings of Quantum Aspects of Gauge Theories, Supersymmetry, and Unification Corfu, Greece - September 1998

\footnotetext{
${ }^{1}$ Work supported in part by the U.S. Dept. of Energy under Grant No. DE-FG03-92-ER40701.
} 


\section{Introduction}

It is a pleasure to speak in such a remarkable venue - the old fortress of Corfu City. It is not often that one gets to meet inside a tourist attraction. The last such occasion for me was at a conference that took place inside the Chateau de Blois.

Many of the talks in this conference will cover recent developments in superstring theory and $M$ theory - in particular, the recent AdS/CFT conjecture. Since the organizers have chosen to place me first in the schedule, and since not everyone here is an expert in these matters, I have decided (in consultation with the organizers) to give a rather general introduction to some of these recent developments. Hopefully, this will help to provide some of the background that is needed for the more specialized talks that will follow. It should also relieve those speakers of the need to give an extended review of the basics.

As I'm sure most of you know, the term M theory was introduced by Witten to describe the 11-dimensional quantum theory whose low energy effective description is 11-dimensional supergravity. However, the usage of this term has been extended by many authors (including myself on occasion) to refer to the underlying theory that reduces to the five different 10dimensional superstring theories in various special limits, as well as the flat 11-dimensional theory in a sixth special limit. This is somewhat confusing. Therefore, in a recent talk at the Vancouver conference, Sen proposed that the term M theory should be reserved for the 11dimensional quantum theory and that the (largely unknown) underlying fundamental theory, which is not specific to any particular spacetime dimension, should be called U Theory [1]. He suggested that U could stand for either "unknown" or "unified". This nomenclature makes a lot of sense to me, so I will try to adhere to it. In the text that follows, I will use the term $\mathrm{M}$ theory and not the term $\mathrm{U}$ theory. However, its usage will occur only in the sense that Witten originally intended, namely an 11-dimensional quantum theory.

In the first half of this talk (in sections $2-5$ ) I will survey some of the basic facts about type IIA and type IIB superstrings in 10 dimensions and the dualities that related them to M theory. Aside from some minor editing, these sections are copied from a review that I wrote earlier this year [2]. Then, in section 6 I will give an introduction to the remarkable duality that has been proposed between superstring theory or M theory in certain anti de Sitter spacetime backgrounds and conformally invariant field theories. To be specific, I will focus on the duality between type IIB superstring theory in an $A d S_{5} \times S^{5}$ background and $\mathcal{N}=4$ supersymmetric gauge theory. As I have already indicated, in this talk I will only survey 
some of the basics, and leave the discussion of more advanced aspects of this subject to other speakers. For a more detailed survey of superstring theory and M theory I recommend the review paper written by Sen [3]. Polchinski's new textbook is also recommended [G].

\section{Perturbative Superstring Theory}

Superstring theory first achieved widespread acceptance during the first superstring revolution in 1984-85. There were three main developments at this time. The first was the discovery of an anomaly cancellation mechanism [5], which showed that supersymmetric gauge theories can be consistent in ten dimensions provided they are coupled to supergravity (as in type I superstring theory) and the gauge group is either $\mathrm{SO}(32)$ or $E_{8} \times E_{8}$. Any other group necessarily would give uncanceled gauge anomalies and hence inconsistency at the quantum level. The second development was the discovery of two new superstring theories - called heterotic string theories - with precisely these gauge groups [6]. The third development was the realization that the $E_{8} \times E_{8}$ heterotic string theory admits solutions in which six of the space dimensions form a Calabi-Yau space, and that this results in a $4 \mathrm{~d}$ effective theory at low energies with many qualitatively realistic features [7]. Unfortunately, there are very many Calabi-Yau spaces and a whole range of additional choices that can be made (orbifolds, Wilson loops, etc.). Thus there is an enormous variety of possibilities, none of which stands out as particularly special.

In any case, after the first superstring revolution subsided, we had five distinct superstring theories with consistent weak coupling perturbation expansions, each in ten dimensions. Three of them, the type $I$ theory and the two heterotic theories, have $\mathcal{N}=1$ supersymmetry in the ten-dimensional sense. Since the minimal 10d spinor is simultaneously Majorana and Weyl, this corresponds to 16 conserved supercharges. The other two theories, called type IIA and type IIB, have $\mathcal{N}=2$ supersymmetry (32 supercharges) [ $[8]$. In the IIA case the two spinors have opposite handedness so that the spectrum is left-right symmetric (nonchiral). In the IIB case the two spinors have the same handedness and the spectrum is chiral.

The understanding of these five superstring theories was developed in the ensuing years. In each case it became clear, and was largely proved, that there are consistent perturbation expansions of on-shell scattering amplitudes. In four of the five cases (heterotic and type II) the fundamental strings are oriented and unbreakable. As a result, these theories have particularly simple perturbation expansions. Specifically, there is a unique Feynman diagram 
at each order of the loop expansion. The Feynman diagrams depict string world sheets, and therefore they are two-dimensional surfaces. For these four theories the unique $L$ loop diagram is a closed orientable genus- $L$ Riemann surface, which can be visualized as a sphere with $L$ handles. External (incoming or outgoing) particles are represented by $N$ points (or "punctures") on the Riemann surface. A given diagram represents a welldefined integral of dimension $6 L+2 N-6$. This integral has no ultraviolet divergences, even though the spectrum contains states of arbitrarily high spin (including a massless graviton). From the viewpoint of point-particle contributions, string and supersymmetry properties are responsible for incredible cancellations. Type I superstrings are unoriented and breakable. As a result, the perturbation expansion is more complicated for this theory, and the various world-sheet diagrams at a given order (determined by the Euler number) have to be combined properly to cancel divergences and anomalies 9].

\section{$2.1 \quad$ T Duality}

An important discovery that was made between the two superstring revolutions is called $T$ duality [10]. This is a property of string theories that can be understood within the context of perturbation theory. (The discoveries associated with the second superstring revolution are mostly nonperturbative.) T duality shows that spacetime geometry, as probed by strings, has some surprising properties (sometimes referred to as quantum geometry). The basic idea can be illustrated by the simplest example in which one spatial dimension forms a circle (denoted $S^{1}$ ). Then the ten-dimensional geometry is $R^{9} \times S^{1}$. T duality identifies this string compactification with one of a second string theory also on $R^{9} \times S^{1}$. However, if the radii of the circles in the two dual descriptions are denoted $R_{1}$ and $R_{2}$, then

$$
R_{1} R_{2}=\alpha^{\prime}
$$

Here $\alpha^{\prime}=\ell_{s}^{2}$ is the universal Regge slope parameter, and $\ell_{s}$ is the fundamental string length scale (for both string theories). The tension of a fundamental string is given by

$$
T=2 \pi m_{s}^{2}=\frac{1}{2 \pi \alpha^{\prime}},
$$

where we have introduced a fundamental string mass scale $m_{s}=\left(2 \pi \ell_{s}\right)^{-1}$.

Note that $\mathrm{T}$ duality implies that shrinking the circle to zero in one theory corresponds to decompactification of the dual theory. Compactification on a circle of radius $R$ implies 
that momenta in that direction are quantized, $p=n / R$. (These are called Kaluza-Klein excitations.) These momenta appear as masses for states that are massless from the higherdimensional viewpoint. String theories also have a second class of excitations, called winding modes. Namely, a string wound $m$ times around the circle has energy

$$
E=2 \pi R \cdot m \cdot T=m R / \alpha^{\prime}
$$

Equation (11) shows that the winding modes and Kaluza-Klein excitations are interchanged under T duality.

What does T duality imply for our five superstring theories? The IIA and IIB theories are $\mathrm{T}$ dual [11]. So compactifying the nonchiral IIA theory on a circle of radius $R$ and letting $R \rightarrow 0$ gives the chiral IIB theory in ten dimensions! This means, in particular, that they should not be regarded as distinct theories. The radius $R$ is actually a vev of a scalar field, which arises as an internal component of the 10d metric tensor. Thus the type IIA and type IIB theories in 10d are two limiting points in a continuous moduli space of quantum vacua. The two heterotic theories are also $\mathrm{T}$ dual, though there are technical details involving Wilson loops, which we will not explain here. T duality applied to the type I theory gives a dual description, which is sometimes called I'. The names IA and IB have also been introduced by some authors.

For the remainder of this paper, we will restrict attention to theories with maximal supersymmetry (32 conserved supercharges). This is sufficient to describe the basic ideas of M theory. Of course, it suppresses many fascinating and important issues and discoveries. In this way we will keep the presentation from becoming too long or too technical. The main focus will be to ask what happens when we go beyond perturbation theory and allow the coupling strength to become large in the type II theories. The answer in the IIA case, as we will see, is that another spatial dimension appears.

\section{$3 \quad$ M Theory}

In the 1970s and 1980s various supersymmetry and supergravity theories were constructed. (See [12], for example.) In particular, supersymmetry representation theory showed that ten is the largest spacetime dimension in which there can be a matter theory (with spins $\leq 1$ ) in which supersymmetry is realized linearly. A realization of this is 10d super Yang-Mills theory, which has 16 supercharges [13]. This is a pretty (i.e., very symmetrical) classical 
field theory, but at the quantum level it is both nonrenormalizable and anomalous for any nonabelian gauge group. However, as we indicated earlier, both problems can be overcome for suitable gauge groups $\left(\mathrm{SO}(32)\right.$ or $\left.E_{8} \times E_{8}\right)$ when the Yang-Mills theory is embedded in a type I or heterotic string theory.

The largest possible spacetime dimension for a supergravity theory (with spins $\leq 2$ ), on the other hand, is eleven. Eleven-dimensional supergravity, which has 32 conserved supercharges, was constructed 20 years ago [14]. It has three kinds of fields - the graviton field (with 44 polarizations), the gravitino field (with 128 polarizations), and a three-index antisymmetric tensor gauge field $C_{\mu \nu \rho}$ (with 84 polarizations). These massless particles are referred to collectively as the supergraviton. 11d supergravity is also a pretty classical field theory, which has attracted a lot of attention over the years. It is not chiral, and therefore not subject to anomaly problems. It is also nonrenormalizable, and thus it cannot be a fundamental theory. (Though it is difficult to demonstrate explicitly that it is not finite as a result of "miraculous" cancellations, we now know that this is not the case.) However, we now believe that it is a low-energy effective description of $M$ theory, which is a welldefined quantum theory [16]. This means, in particular, that higher dimension terms in the effective action for the supergravity fields have uniquely determined coefficients within the M theory setting, even though they are formally infinite (and hence undetermined) within the supergravity context.

\subsection{Relation to Type IIA Superstring Theory}

Intriguing connections between type IIA string theory and 11d supergravity have been known for a long time. If one carries out dimensional reduction of $11 \mathrm{~d}$ supergravity to $10 \mathrm{~d}$, one gets type IIA supergravity [17]. In this case dimensional reduction can be viewed as a compactification on a circle in which one drops all the Kaluza-Klein excitations. It is easy to show that this does not break any of the supersymmetries.

The field equations of 11d supergravity admit a solution that describes a supermembrane. This solution has the property that the energy density is concentrated on a two-dimensional surface. A 3d world-volume description of the dynamics of this supermembrane, quite analogous to the $2 \mathrm{~d}$ world volume actions of superstrings, has been constructed [18]. The authors suggested that a consistent 11d quantum theory might be defined in terms of this membrane,

\footnotetext{
${ }^{2}$ Unless the spacetime has boundaries. The anomaly associated to a $10 \mathrm{~d}$ boundary can be canceled by introducing $E_{8}$ supersymmetric gauge theory on the boundary [15].
} 
in analogy to string theories in ten dimensions. [1 Another striking result was the discovery of double dimensional reduction [19]. This is a dimensional reduction in which one compactifies on a circle, wraps one dimension of the membrane around the circle and drops all Kaluza-Klein excitations for both the spacetime theory and the world-volume theory. The remarkable fact is that this gives the (previously known) type IIA superstring world-volume action [20].

For many years these facts remained unexplained curiosities until they were reconsidered by Townsend 21] and by Witten [16]. The conclusion is that type IIA superstring theory really does have a circular 11th dimension in addition to the previously known ten spacetime dimensions. This fact was not recognized earlier because the appearance of the 11th dimension is a nonperturbative phenomenon, not visible in perturbation theory.

To explain the relation between $\mathrm{M}$ theory and type IIA string theory, a good approach is to identify the parameters that characterize each of them and to explain how they are related. Eleven-dimensional supergravity (and hence $\mathrm{M}$ theory, too) has no dimensionless parameters. As we have seen, there are no massless scalar fields, whose vevs could give parameters. The only parameter is the 11d Newton constant, which raised to a suitable power $(-1 / 9)$, gives the $11 \mathrm{~d}$ Planck mass $m_{p}$. When $\mathrm{M}$ theory is compactified on a circle (so that the spacetime geometry is $R^{10} \times S^{1}$ ) another parameter is the radius $R$ of the circle.

Now consider the parameters of type IIA superstring theory. They are the string mass scale $m_{s}$, introduced earlier, and the dimensionless string coupling constant $g_{s}$. An important fact about all five superstring theories is that the coupling constant is not an arbitrary parameter. Rather, it is a dynamically determined vev of a scalar field, the dilaton, which is a supersymmetry partner of the graviton. With the usual conventions, one has $g_{s}=\left\langle e^{\phi}\right\rangle$.

We can identify compactified M theory with type IIA superstring theory by making the following correspondences:

$$
\begin{aligned}
& m_{s}^{2}=2 \pi R m_{p}^{3} \\
& g_{s}=2 \pi R m_{s} .
\end{aligned}
$$

Using these one can derive other equivalent relations, such as

$$
\begin{gathered}
g_{s}=\left(2 \pi R m_{p}\right)^{3 / 2} \\
m_{s}=g_{s}^{1 / 3} m_{p} .
\end{gathered}
$$

\footnotetext{
${ }^{3}$ It is now clear that this cannot be done in any straightforward manner, since there is no weak coupling limit in which the supermembrane describes all the finite-mass excitations.
} 
The latter implies that the 11d Planck length is shorter than the string length scale at weak coupling by a factor of $\left(g_{s}\right)^{1 / 3}$.

Conventional string perturbation theory is an expansion in powers of $g_{s}$ at fixed $m_{s}$. Equation (3) shows that this is equivalent to an expansion about $R=0$. In particular, the strong coupling limit of type IIA superstring theory corresponds to decompactification of the eleventh dimension, so in a sense $\mathrm{M}$ theory is type IIA string theory at infinite coupling. This explains why the eleventh dimension was not discovered in studies of string perturbation theory.

These relations encode some interesting facts. The fact relevant to eq. (2) concerns the interpretation of the fundamental type IIA string. Earlier we discussed the old notion of double dimensional reduction, which allowed one to derive the IIA superstring world-sheet action from the $11 \mathrm{~d}$ supermembrane (or M2-brane) world-volume action. Now we can make a stronger statement: The fundamental IIA string actually is an M2-brane of M theory with one of its dimensions wrapped around the circular spatial dimension. No truncation to zero modes is required. Denoting the string and membrane tensions (energy per unit volume) by $T_{F 1}$ and $T_{M 2}$, one deduces that

$$
T_{F 1}=2 \pi R T_{M 2}
$$

However, $T_{F 1}=2 \pi m_{s}^{2}$ and $T_{M 2}=2 \pi m_{p}^{3}$. Combining these relations gives eq. (2). It should be emphasized that all the formulas in this section are exact, due to the large amount of unbroken supersymmetry.

\section{2 p-Branes and D-Branes}

Type II superstring theories contain a variety of $p$-brane solutions that preserve half of the 32 supersymmetries. These are solutions in which the energy is concentrated on a p-dimensional spatial hypersurface. (Adding the time dimension, the world volume of a $p$-brane has $p+1$ dimensions.) The corresponding solutions of supergravity theories were constructed by Horowitz and Strominger [22]. A large class of these $p$-brane excitations are called D-branes (or D p-branes when we want to specify the dimension), whose tensions are given by 23]

$$
T_{D p}=2 \pi m_{s}^{p+1} / g_{s}
$$

\footnotetext{
${ }^{4}$ The $E_{8} \times E_{8}$ heterotic string theory is also eleven-dimensional at strong coupling [15].
} 
This dependence on the coupling constant is one of the characteristic features of a D-brane. It is to be contrasted with the more familiar $g^{-2}$ dependence of soliton masses (e.g., the 't Hooft-Polyakov monopole). Another characteristic feature of D-branes is that they carry a charge that couples to a gauge field in the Ramond-Ramond (RR) sector of the theory. (Such fields can be described as bispinors.) The particular RR gauge fields that occur imply that even values of $p$ occur in the IIA theory and odd values in the IIB theory.

D-branes have a number of special properties, which make them especially interesting. By definition, they are branes on which strings can end-D stands for Dirichlet boundary conditions. The end of a string carries a charge, and the D-brane world-volume theory contains a $U(1)$ gauge field that carries the associated flux. When $n \mathrm{D} p$-branes are coincident, or parallel and nearly coincident, the associated $(p+1)$-dimensional world-volume theory is a $U(n)$ gauge theory. The $n^{2}$ gauge bosons $A_{\mu}^{i j}$ and their supersymmetry partners arise as the ground states of oriented strings running from the $i$ th $\mathrm{D} p$-brane to the $j$ th $\mathrm{D} p$-brane. The diagonal elements, belonging to the Cartan subalgebra, are massless. The field $A_{\mu}^{i j}$ with $i \neq j$ has a mass proportional to the separation of the $i$ th and $j$ th branes. This separation is described by the vev of a corresponding scalar field in the world-volume theory.

In particular, the D2-brane of the type IIA theory corresponds to our friend the supermembrane of $\mathrm{M}$ theory, but now in a background geometry in which one of the transverse dimensions is a circle. The tensions check, because (using eqs. (2), (3), and (5))

$$
T_{D 2}=2 \pi m_{s}^{3} / g_{s}=2 \pi m_{p}^{3}=T_{M 2} .
$$

The mass of the first Kaluza-Klein excitation of the $11 \mathrm{~d}$ supergraviton is $1 / R$. Using eq. (3), we see that this can be identified with the D0-brane. More identifications of this type arise when we consider the magnetic dual of the $\mathrm{M}$ theory supermembrane. This turns out to be a five-brane, called the M5-brane. It Its tension is $T_{M 5}=2 \pi m_{p}^{6}$. Wrapping one of its dimensions around the circle gives the D4-brane, with tension

$$
T_{D 4}=2 \pi R T_{M 5}=2 \pi m_{s}^{5} / g_{s}
$$

If, on the other hand, the M5-frame is not wrapped around the circle, one obtains the so-called NS5-brane of the IIA theory with tension

$$
T_{N S 5}=T_{M 5}=2 \pi m_{s}^{6} / g_{s}^{2} .
$$

\footnotetext{
${ }^{5}$ In general, the magnetic dual of a $p$-brane in $d$ dimensions is a $(d-p-4)$-brane.
} 
This 5-brane, which is the magnetic dual of the fundamental IIA string, exhibits the conventional $g^{-2}$ solitonic dependence.

To summarize, type IIA superstring theory is $\mathrm{M}$ theory compactified on a circle of radius $R=g_{s} \ell_{s} . \quad \mathrm{M}$ theory is believed to be a well-defined quantum theory in $11 \mathrm{~d}$, which is approximated at low energy by $11 \mathrm{~d}$ supergravity. Its supersymmetric excitations (which are the only ones known when there is no compactification) are the massless supergraviton, the M2-brane, and the M5-brane. These account both for the (perturbative) fundamental string of the IIA theory and for many of its nonperturbative excitations. The identities presented here are exact, because they are protected by supersymmetry.

\section{Type IIB Superstring Theory}

In the previous section we discussed type IIA superstring theory and its relationship to eleven-dimensional $\mathrm{M}$ theory. In this section we consider type IIB superstring theory, which is the other maximally supersymmetric string theory with 32 conserved supercharges. It is also 10-dimensional, but unlike the IIA theory its two supercharges have the same handedness. Since the spectrum contains massless chiral fields, one should check whether there are anomalies that break the gauge invariances - general coordinate invariance, local Lorentz invariance, and local supersymmetry. In fact, the UV finiteness of the string theory Feynman diagrams (and associated modular invariance) ensures that all anomalies must cancel. This was verified from a field theory viewpoint in ref. [24].

The low-energy effective theory that approximates type IIB superstring theory is type IIB supergravity [8, 25], just as 11d supergravity approximates M theory. In each case the supergravity theory is only well-defined as a classical field theory, but still it can teach us a lot. For example, it can be used to construct $p$-brane solutions and compute their tensions. Even though such solutions themselves are only approximate, supersymmetry ensures that their tensions, which are related to the kinds of charges they carry, are exact.

\section{1 $\mathrm{SL}(2, \mathrm{Z})$ duality}

Another significant fact about type IIB supergravity is that it possesses a global $S L(2, R)$ symmetry. It is instructive to consider the bosonic spectrum and its $S L(2, R)$ transformation properties. There are two scalar fields - the dilaton $\phi$ and an axion $\chi$, which are conveniently 
combined in a complex field

$$
\rho=\chi+i e^{-\phi}
$$

The $S L(2, R)$ symmetry transforms this field nonlinearly:

$$
\rho \rightarrow \frac{a \rho+b}{c \rho+d}
$$

where $a, b, c, d$ are real numbers satisfying $a d-b c=1$. However, in the quantum string theory this symmetry is broken to the discrete subgroup $S L(2, Z)$ [26], which means that $a, b, c, d$ are restricted to be integers. Defining the vev of the $\rho$ field to be

$$
\langle\rho\rangle=\frac{\theta}{2 \pi}+\frac{i}{g_{s}}
$$

the $S L(2, Z)$ symmetry transformation $\rho \rightarrow \rho+1$ implies that $\theta$ is an angular coordinate. More significantly, in the special case $\theta=0$, the symmetry transformation $\rho \rightarrow-1 / \rho$ takes $g_{s} \rightarrow 1 / g_{s}$. This symmetry, called $S$ duality, implies that the theory with coupling constant $g_{s}$ is equivalent to coupling constant $1 / g_{s}$, so that the weak coupling expansion and the strong coupling expansion are identical!

The bosonic spectrum also contains a pair of two-form potentials $B_{\mu \nu}^{(1)}$ and $B_{\mu \nu}^{(2)}$. 9 which transform as a doublet under $S L(2, R)$ or $S L(2, Z)$. In particular, the $\mathrm{S}$ duality transformation $\rho \rightarrow-1 / \rho$ interchanges them. The remaining bosonic fields are the graviton and a four-form potential $C_{\mu \nu \rho \lambda}$, with a self-dual field strength. They are invariant under $S L(2, R)$ or $S L(2, Z)$.

\subsection{Duality Between Type IIB Superstring Theory and M Theory}

In the introductory section we indicated that the type IIA and type IIB superstring theories are $\mathrm{T}$ dual, meaning that if they are compactified on circles of radii $R_{A}$ and $R_{B}$ one obtains equivalent theories for the identification $R_{A} R_{B}=\ell_{s}^{2}$. Moreover, in sect. 2 we saw that the type IIA theory is actually $\mathrm{M}$ theory compactified on a circle. The latter fact encodes nonperturbative information. It turns out to be very useful to combine these two facts and to consider the duality between $\mathrm{M}$ theory compactified on a torus $\left(R^{9} \times T^{2}\right)$ and type IIB superstring theory compactified on a circle $\left(R^{9} \times S^{1}\right)$.

Recall that a torus can be described as the complex plane modded out by the equivalence relations $z \sim z+w_{1}$ and $z \sim z+w_{2}$. Up to conformal equivalence, the periods can be taken

\footnotetext{
${ }^{6}$ These are sometimes denoted $B_{N S}$ and $B_{R R}$.
} 
to be 1 and $\tau$, with $\operatorname{Im} \tau>0$. However, in this characterization $\tau$ and $\tau^{\prime}=(a \tau+b) /(c \tau+d)$, where $a, b, c, d$ are integers satisfying $a d-b c=1$, describe equivalent tori. Thus a torus is characterized by a modular parameter $\tau$ and an $S L(2, Z)$ modular group. The natural, and correct, conjecture at this point is that one should identify the modular parameter $\tau$ of the M theory torus with the parameter $\rho$ that characterizes the type IIB vacuum [27, 28]! Then the duality gives a geometrical explanation of the nonperturbative $\mathrm{S}$ duality symmetry of the IIB theory: the transformation $\rho \rightarrow-1 / \rho$, which sends $g_{s} \rightarrow 1 / g_{s}$ in the IIB theory, corresponds to interchanging the two cycles of the torus in the $\mathrm{M}$ theory description. To complete the story, we should relate the area of the M theory torus $\left(A_{M}\right)$ to the radius of the IIB theory circle $\left(R_{B}\right)$. The desired formula is a simple consequence of the ones given above

$$
m_{p}^{3} A_{M}=\left(2 \pi R_{B}\right)^{-1}
$$

Thus the limit $R_{B} \rightarrow 0$, at fixed $\rho$, corresponds to decompactification of the M theory torus, while preserving its shape. Conversely, the limit $A_{M} \rightarrow 0$ corresponds to decompactification of the IIB theory circle.

The duality can be explored further by matching the various $p$-branes in 9 dimensions that can be obtained from either the $\mathrm{M}$ theory or the IIB theory viewpoints [29]. When this is done, one finds that everything matches nicely and that one deduces various relations among tensions, such as

$$
T_{M 5}=\frac{1}{2 \pi}\left(T_{M 2}\right)^{2}
$$

This relation was used earlier when we asserted that $T_{M 2}=2 \pi m_{p}^{3}$ and $T_{M 5}=2 \pi m_{p}^{6}$.

Even more interesting is the fact that the IIB theory contains an infinite family of strings labeled by pairs of relatively prime integers $(p, q)$ [27]. These integers correspond to string charges that are sources of the gauge fields $B_{\mu \nu}^{(1)}$ and $B_{\mu \nu}^{(2)}$. The $(1,0)$ string can be identified as the fundamental IIB string, while the $(0,1)$ string is the D-string. From this viewpoint, a $(p, q)$ string can be regarded as a bound state of $p$ fundamental strings and $q$ D-strings [30]. These strings have a very simple interpretation in the dual $\mathrm{M}$ theory description. They correspond to an M2-brane with one of its cycles wrapped around a $(p, q)$ cycle of the torus. The minimal length of such a cycle is proportional to $|p+q \tau|$, and thus (using $\tau=\rho$ ) one finds that the tension of a $(p, q)$ string is given by

$$
T_{p, q}=2 \pi|p+q \rho| m_{s}^{2}
$$


The normalization has been chosen to give $T_{1,0}=2 \pi m_{s}^{2}$. Then (for $\left.\theta=0\right) T_{0,1}=2 \pi m_{s}^{2} / g_{s}$, as expected. Note that decay is kinematically forbidden by charge conservation when $p$ and $q$ are relatively prime. When they have a common divisor $n$, the tension is the same as that of an $n$-string system. Whether or not there are threshold bound states is a nontrivial dynamical question, which has different answers in different settings. In the present case there are no such bound states, which is why $p$ and $q$ should be relatively prime.

Imagine that you lived in the 9-dimensional world that is described equivalently as $\mathrm{M}$ theory compactified on a torus or as the type IIB superstring theory compactified on a circle. Suppose, moreover, you had very high energy accelerators with which you were going to determine the "true" dimension of spacetime. Would you conclude that 10 or 11 is the correct answer? If either $A_{M}$ or $R_{B}$ was very large in Planck units there would be a natural choice, of course. But how could you decide otherwise? The answer is that either viewpoint is equally valid. What determines which choice you make is which of the massless fields you regard as "internal" components of the metric tensor and which ones you regard as matter fields. Fields that are metric components in one description correspond to matter fields in the dual one.

\section{The D3-Brane and $\mathcal{N}=4$ Gauge Theory}

The $U(n)$ gauge theory associated with a stack of $n \mathrm{D} p$-branes has maximal supersymmetry (16 supercharges). The low-energy effective theory, when the brane separations are small compared to the string scale, is supersymmetric Yang-Mills theory. These theories can be constructed by dimensional reduction of $10 \mathrm{~d}$ supersymmetric $U(n)$ gauge theory to $p+1$ dimensions. In fact, that is how they originally were constructed [13]. For $p \leq 3$, the low-energy effective theory is renormalizable and defines a consistent quantum theory. For $p=4,5$ there is good evidence for the existence of nongravitational quantum theories that

reduce to the gauge theory in the infrared. For $p \geq 6$, it appears that there is no decoupled nongravitational quantum theory [31].

A case of particular interest, which we shall now focus on, is $p=3$. A stack of $n$ D3-branes in type IIB superstring theory has a decoupled $\mathcal{N}=4, d=4 U(n)$ gauge theory associated to it. This gauge theory has a number of special features. For one thing, due to boson-fermion cancellations, there are no $U V$ divergences at any order of perturbation theory. The beta function $\beta(g)$ is identically zero, which implies that the theory is scale invariant (aside from 
scales introduced by vevs of the scalar fields). In fact, $\mathcal{N}=4, d=4$ gauge theories are conformally invariant. The conformal invariance combines with the supersymmetry to give a superconformal symmetry, which contains 32 fermionic generators. Half are the ordinary linearly realized supersymmetrics, and half are nonlinearly realized ones associated to the conformal symmetry. The name of the superconformal group in this case is $S U(4 \mid 2,2)$. Another important property of $\mathcal{N}=4, d=4$ gauge theories is electric-magnetic duality [32]. This extends to an $S L(2, Z)$ group of dualities. To understand these it is necessary to include a vacuum angle $\theta_{Y M}$ and define a complex coupling

$$
\tau=\frac{\theta_{Y M}}{2 \pi}+i \frac{4 \pi}{g_{Y M}^{2}} .
$$

Under $S L(2, Z)$ transformations this coupling transforms in the usual nonlinear fashion $\left(\tau \rightarrow \frac{a \tau+b}{c \tau+d}\right)$ and the electric and magnetic fields transform as a doublet. Note that the conformal invariance ensures that $\tau$ is a meaningful scale-independent constant.

Now consider the $\mathcal{N}=4 U(n)$ gauge theory associated to a stack of $n$ D3-branes in type IIB superstring theory. There is an obvious identification, that turns out to be correct. Namely, the $S L(2, Z)$ duality of the gauge theory is induced from that of the ambient type IIB superstring theory. In particular, the $\tau$ parameter of the gauge theory is the value of the complex scalar field $\rho$ of the string theory. This makes sense because $\rho$ is constant in the field configuration associated to a stack of D3-branes. The D3-branes themselves are invariant under $S L(2, Z)$ transformations. Only the parameter $\tau=\rho$ changes, but it is transformed to an equivalent value. All other fields, such as $B_{\mu \nu}^{(i)}$, which are not invariant, vanish in this case.

As we have said, a fundamental $(1,0)$ string can end on a D3-brane. But by applying a suitable $S L(2, Z)$ transformation, this configuration is transformed to one in which a $(p, q)$ string — with $p$ and $q$ relatively prime - ends on the D3-brane. The charge on the end of this string describes a dyon with electric charge $p$ and magnetic $q$, with respect to the appropriate gauge field. More generally, for a stack of $n$ D3-branes, any pair can be connected by a $(p, q)$ string. The mass is proportional to the length of the string times its tension, which we saw is proportional to $|p+q \rho|$. In this way one sees that the electrically charged particles, described by fundamental fields, belong to infinite $S L(2, Z)$ multiplets. The other states are nonperturbative excitations of the gauge theory. The field configurations that describe them preserve half of the supersymmetry. As a result their masses saturate a BPS bound and are given exactly by the considerations described above. 


\subsection{Three String Junctions}

An interesting question, whose answer was unknown until recently, is whether $\mathcal{N}=4$ gauge theories in four dimensions also admit nonperturbative excitations that preserve $1 / 4$ of the supersymmetry. To explain the answer, it is necessary to first make a digression to consider three-string junctions.

As we have seen, type IIB superstring theory contains an infinite multiplet of strings labeled by a pair of relatively prime integers $(p, q)$. Three strings, with charges $\left(p_{i}, q_{i}\right)$, $i=1,2,3$, can meet at a point provided that charge is conserved [33, 34]. This means that

$$
\sum p_{i}=\sum q_{i}=0,
$$

if the three strings are all oriented inwards. (This is like momentum conservation in an ordinary Feynman diagram.) Such a configuration is stable, and preserves 1/4 of the ambient supersymmetry provided that the tensions balance. It is easy to see how this can be achieved. If one regards the plane of the junction as a complex plane and orients the direction of a $(p, q)$ string by the phase of $p+q \tau$, then eqs. (11) and (13) ensure a force balance.

The three-string junction has an interesting dual $\mathrm{M}$ theory interpretation. If one of the directions perpendicular to the plane of the junction is taken to be a circle, then we have a string junction in nine dimensions. This must have a dual interpretation in terms of $\mathrm{M}$ theory compactified on a torus. We have already seen that a $(p, q)$ string corresponds to an M2-brane with one of its cycles wrapped on a $(p, q)$ cycle of the torus. So now we join three such cylindrical membranes together. Altogether we have a single smooth M2-brane forming a $Y$, like a junction of pipes. The three arms are wrapped on $\left(p_{i}, q_{i}\right)$ cycles of the torus. This is only possible topologically when eq. (13) is satisfied.

We can now describe a pretty construction of $1 / 4$ BPS states in $\mathcal{N}=4$ gauge theory, due to Bergman [35]. Such a state is described by a 3 -string junction, with the three prongs terminating on three different D3-branes. This is only possible for $n \geq 3$, which is a necessary condition for $1 / 4$ BPS states. The mass of such a state is given by summing the lengths of each string segment weighted by its tension. This gives a result in agreement with the BPS formula. Clearly this is just the beginning of a long story, since the simple picture we have described can be generalized to arbitrarily complicated string webs. So long as the web is in a plane, charges are conserved at the junctions, and all string segments are oriented in the way we have described, the configuration will be 1/4 BPS. Remarkably, arbitrarily high spins 
can occur. There are simple rules for determining them [36]. There are also related results in $\mathcal{N}=2$ (Seiberg-Witten) gauge theory [37, 38]. When the web is nonplanar, supersymmetry is completely broken, and reliable mass calculations become difficult. However, one should still be able to achieve a reliable qualitative understanding of such excitations. In general, there are regions of moduli space in which such nonsupersymmetric states are stable.

\section{Introductory Remarks on AdS/CFT Duality}

Maldacena conjectured a remarkable duality between superstring theory or $\mathrm{M}$ theory in a suitable anti de Sitter space background and conformally invariant field theories [39]. (Some relevant prior papers are listed in ref. [40].) These are dualities in the usual sense: namely, when one description is weakly coupled the corresponding dual one is strongly coupled. Thus, assuming the conjecture is true, it allows the use of perturbative methods to learn nontrivial facts about the strongly coupled dual theory. This subject has developed with breathtaking speed: Maldacena's paper appeared in November 1997, yet by the Strings 98 conference seven months later. more than half the invited speakers chose to speak on this subject. What I propose to do here is to introduce some of the basic ideas of the subject. I will not attempt to be very detailed or precise.

Maldacena arrived at his conjecture by considering the spacetime geometry in the vicinity of a large number $(N)$ of coincident $p$-branes. The three basic examples of AdS/CFT duality with maximal supersymmetry are provided by taking the $p$-branes to be either M2-branes, D3-branes, or M5-branes. Then the corresponding world volume theories (in 3, 4, or 6 dimensions) have superconformal symmetry. They are conjectured to be dual to M theory or type IIB superstring in a spacetime geometry that is $A d S_{4} \times S^{7}, A d S_{5} \times S^{5}$, or $A d S_{7} \times S^{4}$. The background also has nonvanishing gauge fields with $N$ units of flux on the sphere. All three of these solutions to $11 \mathrm{~d}$ supergravity or type IIB supergravity were studied over a decade ago 41, 42, 43, but the duality conjecture is new.

Since I am not trying to be comprehensive, only the case of coincident D3 branes will be described. However, in order to explain what is special about the case $p=3$, we will begin by considering $N$ coincident $\mathrm{D} p$-branes. This is a type IIA configuration if $p$ is even and a type IIB configuration if $p$ is odd. As we have discussed, the $(p+1)$-dimensional worldvolume theory (in the infrared) is a maximally supersymmetric $U(N)$ gauge theory. The

\footnotetext{
${ }^{7}$ All the talks (including audio) are available at http://www.itp.ucsb.edu $\sim$ strings $98 /$.
} 
low-energy effective action is given by dimensional reduction of supersymmetric $U(N)$ gauge theory in 10 dimensions. The coupling constant $g_{Y M}$ of such a gauge theory has dimensions (length) ${ }^{(p-3) / 2}$. It is related to the dimensionless string coupling constant $g_{s}$ of the ambient 10d theory by

$$
g_{Y M}^{2}=g_{s}\left(\ell_{s}\right)^{p-3}
$$

Of course, the dimensionless effective coupling is scale dependent. At an energy scale $E$ it is given by

$$
g_{\text {eff }}^{2}(E)=g_{Y M}^{2} N E^{p-3}
$$

Thus perturbation theory applies in the UV for $p<3$ and in the IR for $p>3$. The special case $p=3$ corresponds to $\mathcal{N}=4$ super Yang-Mills theory in four dimensions, which is known to be a finite, conformally invariant field theory.

As solutions of type II supergravity, an extremal system of $N$ coincident $\mathrm{D} p$-branes has a string-frame metric

$$
d s^{2}=f^{-1 / 2}\left(d s^{2}\right)_{d}+f^{1 / 2}\left(d s^{2}\right)_{10-d},
$$

dilaton

$$
e^{2 \phi}=g_{s}^{2}(f)^{\frac{3-p}{2}}
$$

and RR gauge field

$$
C_{01 \ldots p}=f^{-1}-1
$$

where $d=p+1$ and

$$
\begin{gathered}
\left(d s^{2}\right)_{d}=-d t^{2}+d x_{1}^{2}+\ldots+d x_{p}^{2} \\
\left(d s^{2}\right)_{10-d}=d x_{p+1}^{2}+\ldots+d x_{9}^{2}=d r^{2}+r^{2} d \Omega_{8-p}^{2} \\
f=1+\frac{g_{Y M}^{2} N}{\ell_{s}^{4} U^{7-p}} \\
U=r / \ell_{s}^{2} .
\end{gathered}
$$

The variable $U \sim T r$ is essentially the energy of a string stretched between the D-branes at $r=0$ and a point a distance $r$ from the D-branes. The surface $r=0$ is the horizon of this geometry, which can be regarded as the location of the D-branes.

The key step in Maldacena's analysis is to isolate the behavior of the near-horizon geometry by letting $\ell_{s} \rightarrow 0$ while holding $U$ fixed. In this limit one finds that

$$
d s^{2} \rightarrow \ell_{s}^{2}\left\{\frac{1}{\sqrt{\lambda}} U^{\frac{7-p}{2}}\left(d x^{2}\right)_{d}+\sqrt{\lambda} U^{\frac{p-7}{2}} d U^{2}+\sqrt{\lambda} U^{\frac{p-3}{2}} d \Omega_{8-p}^{2}\right\},
$$


where

$$
\lambda=g_{Y M}^{2} N
$$

Also,

$$
e^{\phi} \rightarrow \frac{1}{N}\left[g_{e f f}(U)\right]^{\frac{7-p}{2}} .
$$

From these equations we see that the string is weakly coupled for $g_{\text {eff }}^{2}(U) \ll N^{4 /(7-p)}$ and that the curvature is of order $\left[\ell_{s}^{2} g_{e f f}(U)\right]^{-1}$. Thus the supergravity approximation is good (i.e., stringy effects are negligible) for $g_{\text {eff }}^{2}(U) \gg 1$. Taking $p<6$ and requiring both of these inequalities gives the requirement $N \gg 1$. There is much more that can be said for each specific value of $p$. However, we will focus on the special case $p=3$ from now on, and refer the reader to ref. 444 for a discussion of the other cases.

Taking $p=3$, the near-horizon metric in eq. (17) simplifies to

$$
d s^{2}=\ell_{s}^{2}\left\{\frac{1}{\sqrt{\lambda}} U^{2}\left(d x^{2}\right)_{4}+\frac{\sqrt{\lambda}}{U^{2}} d U^{2}+\sqrt{\lambda} d \Omega_{5}^{2}\right\},
$$

and the dilaton is constant:

$$
e^{\phi}=g_{s}
$$

Making the change of variables $z=\sqrt{\lambda} / U$, the metric takes the form

$$
d s^{2}=\ell_{s}^{2} \sqrt{\lambda}\left\{\frac{\left(d x^{2}\right)_{4}+d z^{2}}{z^{2}}+d \Omega_{5}^{2}\right\} .
$$

This describes the product-space geometry $A d S_{5} \times S^{5}$, where both factors have radius

$$
R=\lambda^{1 / 4} \ell_{s}
$$

We see that stringy effects are suppressed for $\lambda \gg 1$. Quantum corrections are small for $N \gg 1$, since the Planck length is given by $\ell_{p}=g_{s}^{1 / 4} \ell_{s}$ and $\lambda=g_{s} N$. The value of the RR gauge field corresponds to the self-dual field strength five-form

$$
F_{5} \sim N\left((\operatorname{vol})_{A d S_{5}}+(\operatorname{vol})_{S^{5}}\right)
$$

In particular the flux $\int_{S^{5}} F_{5} \sim N$. The $A d S_{5} \times S^{5}$ metric has isometries

$$
S O(4,2) \times S O(6) \approx S U(2,2) \times S U(4) .
$$

Including the supersymmetries, the complete isometry supergroup is $S U(2,2 \mid 4)$. This contains 32 supercharges transforming as $(4,4)+(\overline{4}, \overline{4})$. 


\subsection{The Conjecture}

Maldacena's conjecture is that type IIB superstring theory in the $A d S_{5} \times S^{5}$ background described above is dual to $\mathcal{N}=4, d=4$ super Yang-Mills theory with gauge group $S U(N)$. This is plausible because this theory is associated to a system of $N$ coincident D3-branes, as we have explained. The passage from $U(N)$ to $S U(N)$ is a technical detail that I will not attempt to explain. The duality incorporates the following correspondences:

- The (large) integer $N$ gives the rank of the gauge group, which corresponds to the flux of the five-form RR gauge field.

- The YM coupling constant $g_{Y M}$ is related to the string coupling constant by $g_{Y M}^{2}=g_{s}$. The fact that $g_{Y M}$ does not depend on energy scale corresponds to the fact the dilaton is constant.

- The supergroup $\mathrm{SU}(2,2 \mid 4)$ is the isometry group of the superstring theory background and it is the superconformal symmetry group of the $\mathcal{N}=4$ gauge theory. In the gauge theory, 16 of the fermionic generators are linearly realized supersymmetries and the other 16 generate superconformal transformations.

- The common radius $R$ of the $A d S_{5}$ and $S^{5}$ geometries is related to the 't Hooft parameter $\lambda=g_{Y M}^{2} N$ of the gauge theory by $R / \ell_{s}=\lambda^{1 / 4}$.

As a side remark, let me point out that the $A d S_{5} \times S^{5}$ metric is conformally flat, because the two radii are equal. As a result, in addition to its isometries it has additional conformal isometries. From the point of view of the dual gauge theory these correspond to the 2410 dimensional Lorentz transformations that are broken by dimensional reduction from $d=10$ to $d=4$. They have no analogs for the M theory backgrounds $A d S_{4} \times S^{7}$ and $A d S_{7} \times S^{4}$, because the radii are unequal in these cases.

\subsection{The Structure of Anti de Sitter Space}

In the preceding we have presented $A d S_{5}$ in Poincaré coordinates. In these coordinates, $A d S_{d+1}$ is given by

$$
d s^{2}=\frac{1}{z^{2}}\left(\left(d x^{2}\right)_{d}+d z^{2}\right), \quad z \geq 0 .
$$

\footnotetext{
${ }^{8}$ For a discussion of this point, and how it is reconciled with the $\mathrm{SL}(2, \mathrm{Z})$ duality, see ref. 45.
} 
The boundary at spatial infinity $(r \rightarrow \infty)$ corresponds to $z=0$. The AdS/CFT duality is holographic in the sense that the physics of the $(d+1)$-dimensional bulk is encoded in the $d$-dimensional boundary gauge theory. But how does this hologram work? The basic idea is that $x^{\mu}$ coordinates of a point in the bulk correspond to the $x^{\mu}$ position in the field theory, whereas the $z=\sqrt{\lambda} / U$ coordinate corresponds to taking the field theory to have an energy scale (in the Wilsonian sense) $E=U$.

One fact in support of the identification $E \propto U$ is that the bulk isometry $\left(x^{\mu}, z\right) \rightarrow$ $\left(a x^{\mu}, a z\right)$ corresponds to the field theory scale transformation $\left(x^{\mu}, E\right) \rightarrow\left(a x^{\mu}, E / a\right)$. This argument does not establish the constant of proportionality, however. An argument that achieves this is the identification of D-instantons in the bulk with YM instantons of the gauge theory. It turns out that the $z$ coordinate of the D instanton corresponds to the scale size of the YM instanton. This suggests the identification $U=\sqrt{\lambda} E$. It is a somewhat puzzling fact that this argument gives the factor of $\sqrt{\lambda}$, whereas the "stretched string" picture, discussed earlier, does not 46].

Poincaré coordinates do not give a complete description of the Lorentzian $A d S_{d+1}$ spacetime. To understand this, it is useful to consider a hypersurface in $d+2$ Euclidean dimensions:

$$
x_{1}^{2}+\ldots+x_{d}^{2}-t_{1}^{2}-t_{2}^{2}=-R^{2}=-1 \text {. }
$$

In the last step the radius has been set equal to one, for convenience. Next, we pass to spherical coordinates for both the $x$ 's and the $t$ 's:

$$
\begin{gathered}
\left(x_{1}, \ldots, x_{d}\right) \rightarrow\left(r, \Omega_{p}\right) \\
\left(t_{1}, t_{2}\right) \rightarrow(\tau, \theta) .
\end{gathered}
$$

In these coordinates the hypersurface is $r^{2}-\tau^{2}=-1$, and the metric on this surface is

$$
d s^{2}=\sum d x_{i}^{2}-\sum d t_{j}^{2}=\frac{d r^{2}}{1+r^{2}}+r^{2} d \Omega_{p}^{2}-\left(1+r^{2}\right) d \theta^{2}
$$

Note that the time-like coordinate $\theta$ is periodic! This would imply that the conjugate energy eigenvalues are quantized. This is definitely not what type IIB superstring theory on $A d S_{5} \times S^{5}$ gives, so we must pass to the covering space CAdS. Strictly speaking, one should speak of "CAdS/CFT duality." So we replace $\theta \in S^{1}$ by $t \in R$. This gives a global description of the desired spacetime geometry. Letting $r=\tan \rho$, the metric becomes

$$
d s^{2}=\frac{1}{\cos ^{2} \rho}\left(d \rho^{2}+\sin ^{2} \rho d \Omega_{p}^{2}-d t^{2}\right)
$$


This has topology $B_{p+1} \times R$ which can be visualized as a solid cylinder. The $R$ factor corresponds to the global time coordinate $t$, and $B_{p+1}$ is the ball interior to $S^{p}$. The boundary of the spacetime (at $\rho=\pi / 2$ ) is $S^{p} \times R$. Thus the spatial coordinates of the dual gauge theory should be compactified on a sphere $S^{p}$. (The conformal symmetry also requires adjoining the point at infinity.) The $S^{p} \times R$ geometry makes the $S O(p+1) \times S O(2)$ subgroup of the $S O(p+1,2)$ conformal group manifest. Because of the conformal invariance, the radius of the $S^{p}$ is unphysical - there is no scale. One recovers Minkowski spacetime by letting it become infinite. The relation between the coordinates introduced here and the Poincaré coordinates given earlier is

$$
\left(z, y^{\mu}\right)=\left(\left(t_{1}+x_{d}\right)^{-1}, t_{2} z, x_{i} z\right)
$$

For many purposes it is useful to consider the "Euclideanized" AdS geometry. This can be obtained by Wick-rotating the $t_{2}$ coordinate:

$$
x_{1}^{2}+\ldots+x_{d}^{2}-t_{1}^{2}+t_{2}^{2}=-1
$$

The symmetry is now $S O(1, d+1)$. This manifold should not be confused with Lorentzian signature de Sitter space, which would have +1 on the right-hand side.

As before, this manifold can be described in Poincaré coordinates by

$$
d s^{2}=\frac{1}{z^{2}}\left(d z^{2}+\left(d y^{2}\right)_{d}\right)
$$

where now

$$
\left(d y^{2}\right)_{d}=d y_{1}^{2}+\ldots+d y_{d}^{2}
$$

Unlike the Lorentzian case, these coordinates describe the space globally. They give a description that is equivalent to the one given by the metric

$$
d s^{2}=d r^{2}+\sinh ^{2} r d \Omega_{d}^{2}
$$

which is the analog of eq. (23). Another equivalent metric is

$$
d s^{2}=\frac{4 \sum_{i=1}^{d+1} x_{i}^{2}}{\left(1-\sum x_{i}^{2}\right)^{2}},
$$

where $\sum x_{i}^{2} \leq 1$. The latter form shows that the topology is that of a ball $B_{d+1}$ whose boundary is a sphere $S^{d}$. Thus the dual Euclideanized gauge theory should be compactified on a sphere $-S^{4}$ for our main example. In this case the $\mathrm{SO}(5)$ subgroup of the $\mathrm{SO}(5,1)$ 
conformal group is manifest. Of course, conformal symmetry allows one to let the radius go to infinity.

The AdS/CFT conjecture has been made more precise by Gubser, Klebanov, and Polyakov [47] and by Witten [48]. They give an explicit prescription for relating correlation functions of the Euclideanized conformal field theory to the bulk theory path integral for specified boundary behavior of the bulk fields. I will not spell out the prescription carefully here, but simply remark that it requires a one-to-one correspondence of bulk fields $\phi$ and gauge invariant boundary operators $\mathcal{O}$. Denoting boundary values of $\phi$ by $\phi_{0}$, one computes the bulk theory path integral with these boundary values $Z\left(\phi_{0}\right)$. Then this is identified with the correlation function $\left\langle\exp \int_{S^{d}} \phi_{0} \mathcal{O}\right\rangle_{C F T}$. (I am ignoring lots of technical details here.) The requisite correspondences have been verified for large classes of examples.

The CAdS/CFT duality for Lorentzian signature entails new issues that have been considered in ref. 49. The boundary value problem in this case no longer has unique solutions, because one can add normalizable (propagating) modes. The conclusion of 49, if I understand it properly, is that nonnormalizable bulk modes correspond to backgrounds that couple to gauge invariant local operators of the boundary gauge theory, as in the Euclidean case. In addition, the normalizable modes correspond to localized fluctuations of the gauge theory. The latter do not appear in the Euclidean case, so this identification goes beyond those proposed in refs. [47, 48].

\subsection{Finite Temperature}

The passage to finite temperature is evident. One starts with the Euclideanized theory described above and takes the time coordinate to have period $\beta$, the inverse temperature. Fermi fields are required to be antiperiodic, as usual, which breaks supersymmetry. The topology of the boundary CFT in this case is $S^{p} \times S^{1}$. Witten observes that there are two different choices for the topology of the bulk that would give this boundary [50]. The one suggested by the zero-temperature analysis is $B_{p+1} \times S^{1}$ and an alternative possibility is $S^{p} \times B_{2}$. The first choice corresponds to $A d S_{d+1}$ at finite temperature and the second to a (Euclidean) AdS-Schwarzschild black hole. By comparing the action for the two, Witten argues that there is a phase transition for $N \rightarrow \infty$. The low temperature phase exhibits confinement and a mass gap, whereas the high temperature phase has deconfinement. This is roughly the picture one expects for QCD. 


\section{Concluding Comments}

I have touched on some of the highlights in the remarkable development of superstring theory that has taken place in the past few years. It continues to amaze me that the rapid pace of progress is being maintained over such an extended period. Certainly, the implications of the AdS/CFT duality are still being digested, so it will continue for a while longer. In the brief introduction to this topic in the preceding section, I have not mentioned many of the applications and generalizations that have already been worked out. This work already makes it clear that this duality will teach us a great deal about strongly coupled gauge theories - in particular their large $\mathrm{N}$ master fields. It is less clear, but likely, that it will also enhance our understanding of nonperturbative string theory. Even so, I have the feeling that qualitatively new insights are still required to properly address the issue of the cosmological constant and the stabilization of moduli.

\section{References}

[1] A. Sen, hep-ph/9810356.

[2] J. H. Schwarz, hep-th/9807135.

[3] A. Sen, hep-th/9802051.

[4] J. Polchinski, String Theory in 2 vols., Cambridge U. Press (1998).

[5] M.B. Green and J.H. Schwarz, Phys. Lett. 149B (1984) 117.

[6] D.J. Gross, J.A. Harvey, E. Martinec, and R. Rohm, Phys. Rev. Lett. 54 (1985) 502.

[7] P. Candelas, G.T. Horowitz, A. Strominger, and E. Witten, Nucl. Phys. B258 (1985) 46.

[8] M.B. Green and J.H. Schwarz, Phys. Lett. 109B (1982) 444.

[9] M.B. Green and J.H. Schwarz, Phys. Lett. 151B (1985) 21; Nucl. Phys. B255 (1985) 93.

[10] For a review see A. Giveon, M. Porrati, and E. Rabinovici, Phys. Rept. 244 (1994) 77, hep-th/9401139. 
[11] P. Ginsparg, Phys. Rev. D35 (1987) 648; M. Dine, P. Huet, and N. Seiberg, Nucl. Phys. B322 (1989) 301.

[12] A. Salam and E. Sezgin, eds., Supergravities in Diverse Dimensions, reprints in 2 vols., World Scientific (1989).

[13] L. Brink, J.H. Schwarz, and J. Scherk, Nucl. Phys. B121 (1977) 77; F. Gliozzi, J. Scherk, and D. Olive, Nucl. Phys. B122 (1977) 253.

[14] E. Cremmer, B. Julia, and J. Scherk, Phys. Lett. 76B (1978) 409.

[15] P. Hořava and E. Witten, Nucl. Phys. B460 (1996) 506, hep-th/9510209.

[16] E. Witten, Nucl. Phys. B443 (1995) 85, hep-th/9503124.

[17] C. Campbell and P. West, Nucl. Phys. B243 (1984) 112; M. Huq and M. Namazie, Class. Quantum Grav. 2 (1985) 293; F. Giani and M. Pernici, Phys. Rev. D30 (1984) 325.

[18] E. Bergshoeff, E. Sezgin, and P.K. Townsend, Phys. Lett. B189 (1987) 75.

[19] M.J. Duff, P.S. Howe, T. Inami, and K.S. Stelle, Phys. Lett. B191 (1987) 70.

[20] M.B. Green and J.H. Schwarz, Phys. Lett. 136B (1984) 367.

[21] P.K. Townsend, Phys. Lett. B350 (1995) 184, hep-th/9501068.

[22] G.T. Horowitz and A. Strominger, Nucl. Phys. B360 (1991) 197.

[23] J. Polchinski, Phys. Rev. Lett. 75 (1995) 4724, hep-th/9510017; p. 293 in Fields, Strings, and Duality (TASI 96), eds. C. Efthimiou and B. Greene, World Scientific 1997, hep-th/9611050.

[24] L. Alvarez-Gaumé and E. Witten, Nucl. Phys. B234 (1983) 269.

[25] J.H. Schwarz and P.C. West, Phys. Lett. 126B (1983) 301; J.H. Schwarz, Nucl. Phys. B226 (1983) 269; P. Howe and P. West, Nucl. Phys. B238 (1984) 181.

[26] C. Hull and P. Townsend, Nucl. Phys. B438 (1995) 109, hep-th/9410167. 
[27] J.H. Schwarz, Phys. Lett. B360 (1995) 13, Erratum: Phys. Lett. B364 (1995) 252, hep-th/9508143.

[28] P.S. Aspinwall, Nucl. Phys. Proc. Suppl. 46 (1996) 30, hep-th/9508154.

[29] J.H. Schwarz, Phys. Lett. B367 (1996) 97, hep-th/9510086.

[30] E. Witten, Nucl. Phys. B460 (1996) 335, hep-th/9510135.

[31] A. Sen, hep-th/9709220; N. Seiberg, Phys. Rev. Lett. 79 (1997) 3577, hep-th/9710009.

[32] C. Montonen and D. Olive, Phys. Lett. B72 (1977) 117; P. Goddard, J. Nuyts, and D. Olive, Nucl. Phys. B125 (1977) 1; H. Osborne, Phys. Lett. B83 (1979) 321.

[33] O. Aharony, J. Sonnenschein, and S. Yankielowicz, Nucl. Phys. B474 (1996) 309, hep-th/9603009.

[34] J.H. Schwarz, p. 359 in Fields, Strings, and Duality (TASI 96), eds. C. Efthimiou and B. Greene, World Scientific 1997, hep-th/9607201.

[35] O. Bergman, Nucl. Phys. B525 (1998) 104, hep-th/9712211.

[36] O. Bergman and B. Kol, hep-th/9804160.

[37] O. Bergman and A. Fayyazuddin, Nucl. Phys. B531 (1998) 108, hep-th/9802033 and Nucl. Phys. B535 (1998) 139, hep-th/9806011; O. Bergman, hep-th/9811064.

[38] M.R. Gaberdiel, T. Hauer and B. Zwiebach, Nucl. Phys. B525 (1998) 117, hepth/9801205 ; O. DeWolfe, T. Hauer, A. Iqbal, and B. Zwiebach, Nucl. Phys. B534 (1998) 261, hep-th/9805220.

[39] J.M. Maldacena, Adv. Theor. Math. Phys. 2 (1998) 231, hep-th/9711200.

[40] I.R. Klebanov, Nucl. Phys. B496 (1997) 231, hep-th/9702076; S.S. Gubser, I.R. Klebanov, and A.A. Tseytlin, Nucl. Phys. B499 (1997) 217, hep-th/9703040; S.S. Gubser and I.R. Klebanov, Phys. Lett. B413 (1997) 41, hep-th/9708005; A.M. Polyakov, Nucl. Phys. Proc. Suppl. 68 (1998) 1, hep-th/9711002.

[41] P. Freund and M. Rubin, Phys. Lett. 97B (1980) 233; for a review see M.J. Duff, B.E.W. Nilsson, and C.N. Pope, Phys. Rept. 130 (1986) 1. 
[42] K. Pilch, P. van Nieuwenhuizen, and P.K. Townsend, Nucl. Phys. B242 (1984) 377.

[43] H.J. Kim, L.J. Romans, and P. van Nieuwenhuizen, Phys. Rev. D32 (1985) 389; M. Günaydin and N. Marcus, Class. Quant. Grav. 2 (1985) L11.

[44] N. Itzhaki, J. M. Maldacena, J. Sonnenschein, and S. Yankielowicz, Phys. Rev. D58 (1998) 046004, hep-th/9802042.

[45] E. Witten, hep-th/9812012.

[46] A.W. Peet and J. Polchinski, hep-th/9809022.

[47] S.S. Gubser, I.R. Klebanov, and A.M. Polyakov, Phys. Lett. B428 (1998) 105, hepth/9802109;

[48] E. Witten, Adv. Theor. Math. Phys. 2 (1998) 253, hep-th/9802150.

[49] V. Balasubramanian, P. Kraus, and A. Lawrence, hep-th/9805171.

[50] E. Witten, Adv. Theor. Math. Phys. 2 (1998) 505, hep-th/9803131. 